\title{
A structure theorem for operators with closed range
}

\section{James Guyker}

A characterization has previously been given for linear transformations in Hilbert space whose first $N+1$ powers are partial isometries. An analogous characterization is now obtained for transformations whose first $N+1$ powers have closed ranges. A hypothesis (that transformations have no isometric part) is found to be unnecessary in previous work.

\section{Introduction}

A (closed) subspace $M$ of a Hilbert space $H$ is said to reduce a continuous linear transformation $T$ on $H$ if $M$ is invariant under both $T$ and $T^{*}$. The operator $T$ is a partial isometry if $\|T f\|=\|f\|$ for every vector $f$ in $H$ which is orthogonal to the kernel of $T$, or equivalently, if $T=T T^{*} T,[7]$.

In [8], Halmos and Wallen showed that every partial isometry all of whose positive integral powers are partial isometries is a unique direct sum of unitary operators, pure isometries, pure co-isometries, and truncated shifts, with each type of summand (each index for a truncated shift) occurring at most once. Using [6] and the canonical model of de Branges, Rovnyak [2], an explicit description of the reducing subspaces of partial isometries $T$ such that $T^{2}, T^{3}, \ldots, T^{N+1}$ are partial isometries was obtained in [5] under the assumption that $T$ has no isometric part, that is, there is no nonzero vector $f$ in $H$ such that $\left\|T^{n} f\right\|=\|f\|$ for

Received 15 November 1977. The author wishes to express his appreciation to Louis de Branges for his helpful suggestions concerning this paper. 
every $n=1,2, \ldots$. This resulted in a characterization of such partial isometries as unique direct sums of truncated shifts $T_{j}$ of index $j$ $(j=1,2, \ldots, N)$ and partial isometries $V$ with no isometric part whose range includes the kernel of $V^{N}$, with each type of summand occurring at most once, thereby extending the Halmos-Wallen Theorem.

In the present paper the problem of obtaining similar results for operators $T$ with closed range whose first $N+1$ powers have closed range is considered. Under the stronger assumptions that the kernels of $T^{j}$ and $T^{* j}$ are invariant under $T T^{*}$ and $T^{*} T$, respectively, for every $j=1,2, \ldots, N$, the above structure theorem is shown to hold essentially for $T$. A slightly more general concept of truncated shift is necessary, although these generalized shifts enjoy many of the basic properties of truncated shifts.

If $F$ is a family of subsets of $H$, then $v\{F: F \in F\}$ will denote the closed span of the union $U\{F: F \in F\}$. For subspaces $M$ and $N$ of $H$, if $M \perp N$, then $M \oplus N$ will denote the orthogonal direct sum of $M$ and $N$; if $N \subseteq M$, then $M \ominus N$ will be the orthogonal complement of $N$ in $M$.

\section{Reducing subspaces of generalized truncated shifts}

We recall that for a given Hilbert space $H_{0}$ and integer $N \geq 1$, a truncated shift of index $N$ is an operator $T_{N}$ on the direct sum $H_{0} \oplus H_{0} \oplus \ldots \oplus H_{0}$ of $N$ copies of $H_{0}$ which is defined by $T_{N}\left(e_{1}, e_{2}, \ldots, e_{N}\right)=\left(e_{2}, e_{3}, \ldots, e_{N}, 0\right),[8]$. If $A_{1}, A_{2}, \ldots, A_{N}$ are operators on $H_{0}$, the diagonal operator $D=\left(A_{1}, A_{2}, \ldots, A_{N}\right)$ on $H_{0} \oplus H_{0} \oplus \ldots \oplus H_{0}$ is given by

$$
D\left(e_{1}, e_{2}, \ldots, e_{N}\right)=\left(A_{1} e_{1}, A_{2} e_{2}, \ldots, A_{N} e_{N}\right) .
$$

An operator $T$ on a Hilbert space $H$ will be called a generalized truncated shift of index $N$ if the images $T^{* j}$ ker $T(j=0,1, \ldots, N-1)$ form a family of pairwise orthogonal (closed) subspaces which spans $H$ such that $T T^{* j}$ ker $T=T^{* j-1}$ ker $T$ for every $j=1,2, \ldots, N-1$. 
THEOREM 2.1. An operator $T$ on a Hilbert space $H$ is a generalized truncated shift of index $N \geq 2$ if and only if there exist invertible operators $A_{1}, A_{2}, \ldots, A_{N-1}$ defined on a Hilbert space $H_{0}$ such that $T$ is unitarily equivalent to the weighted truncated shift $D T_{N}$ on $H_{0} \oplus H_{0} \oplus \ldots \oplus H_{0}$, where $D=\left(A_{1}, A_{2}, \ldots, A_{N-1}, 0\right)$ and $T_{N}$ is a truncated shift of index $N$.

Proof. A direct computation shows that every weighted truncated shift with invertible weights is a generalized truncated shift of the same index.

Conversely, let $T$ be a generalized truncated shift of index $N$. Since $\operatorname{ker} T=T^{j} T^{* j}$ ker $T$, there exists a unitary operator $U_{j}: \operatorname{ker} T \rightarrow T^{* j}$ ker $T$ for each $j=1,2, \ldots, N-1$. Define the unitary operator

$$
U: \operatorname{ker} T \oplus \operatorname{ker} T \oplus \ldots \oplus \operatorname{ker} T \rightarrow H
$$

by $U\left(e_{1}, e_{2}, \ldots, e_{N}\right)=\left(e_{1}, U_{1} e_{2}, U_{2} e_{3}, \ldots, U_{N-1} e_{N}\right)$. The operator $A_{j}=U_{j-1}^{-1} T U_{j}$, where $U_{0}=I$, defined on the kernel of $T$ for every $j=1,2, \ldots, N-1$, is invertible, and $U^{-1} T U=D T_{N}$ where $D=\left(A_{1}, A_{2}, \ldots, A_{N-1}, 0\right)$.

COROLLARY 2.1. The adjoint of a generalized truncated shift is a generalized truncated shift of the same index.

Proof. The adjoint of $D T_{N}$ where $D=\left(A_{1}, A_{2}, \ldots, A_{N-1}, 0\right)$ as in Theorem 2.1 is unitarily equivalent to $D^{\prime} T_{N}$ where $D^{\prime}=\left(A_{N-1}^{*}, A_{N-2}^{*}, \ldots, A_{1}^{*}, 0\right)$.

COROLLARY 2.2. The only generalized truncated shifts of index $N$ that are partial isometries are the truncated shifts of index $N$.

Proof. Let $T$ be a partially isometric generalized truncated shift of index $N$. For each $j=1,2, \ldots, N-1$, define the operator $U_{j}: \operatorname{ker} T+T^{* j}$ ker $T$ by $U_{j} f=T^{* j} f$ for $f$ in the kernel of $T$. Then 
$U_{j}$ is unitary for every $j$, since $T^{* j-1}$ ker $T$ is contained in the range of $T$, and hence, for every vector $f$ in the kernel of $T$,

$$
\left\|U_{j} f\right\|=\left\|T^{*}\left(T^{* j-1} f\right)\right\|=\left\|T^{*}\left(T^{* j-2} f\right)\right\|=\ldots=\|f\|
$$

for every $j=1,2, \ldots, N-1$.

As in the proof of Theorem 2.1, $T$ is unitarily equivalent to $D T_{N}$, where $D=\left(A_{1}, A_{2}, \ldots, A_{N-1}, 0\right)$ and $A_{j}=U_{j-1}^{-1} T U_{j}\left(U_{0}=I\right)$. Let $f$ be in the kernel of $T$. Since $T^{*^{j-1}} f$ is in the range of $T$, we have that

$$
A_{j} f=U_{j-1}^{-1} T U_{j} f=U_{j-1}^{-1} T T^{*}\left(T^{* j-1} f\right)=U_{j-1}^{-1} T^{* j-1} f=U_{j-1}^{-1} U_{j-1} f=f
$$

for every $j=1,2, \ldots, N-1$. Therefore $A_{j}=I$ for every $j$ and $D T_{N}=T_{N}$

The next result characterizes the reducing subspace structure of generalized truncated shifts.

THEOREM 2.2. Let $T$ be an operator on a Hilbert space $H$ such that the kernel of $T$ is invariant under $T^{k} T^{*}$ for every $k=1,2, \ldots, N-1$, and

$$
H=v\left\{T^{*} \text { ker } T: n=0, I, \ldots, N-1\right\} .
$$

A subspace $M$ of $H$ reduces $T$ if and only if

$$
M=v\left\{T^{*}{ }^{n}: n=0,1, \ldots, N-1\right\}
$$

for some unique subspace $S$ of the kernel of $T$ which is invariant under $T^{k} T^{*}$ for every $k=1,2, \ldots, N-1$. In this case,

$$
H \ominus M=v\left\{T^{*}{ }^{n}(\operatorname{ker} T \Theta S): n=0,1, \ldots, N-1\right\} \text {. }
$$

Proof. Suppose $M$ reduces $T$. Let $P$ be the (orthogonal) projection of $H$ onto $M$. Then

$$
M=P H=\vee\left\{T^{*}{ }^{n} \text { ker } T: n=0,1, \ldots, N-1\right\},
$$


and $S=P$ ker $T \subseteq$ ker $T$ is closed and invariant under $T^{k} T^{k}$ for every $k=1,2, \ldots, N-1$.

The form of $H \Theta M$ is obtained similarly, since $H \Theta M=(1-P) H$ and ker $T=P$ ker $T \oplus(1-P)$ ker $T$.

Conversely, suppose $M$ is of the above form. For every $k, n=0,1, \ldots, N-1, T^{* k} S$ is orthogonal to $T^{*} n(\operatorname{ker} T \Theta S)$. Since $T^{* N}=0$ and

$$
H=v\left\{T^{*} \text { ker } T: n=0,1, \ldots, N-1\right\},
$$

it follows that

$$
H \Theta M=v\left\{T^{*}{ }^{n}(\operatorname{ker} T \Theta S): n=0,1, \ldots, N-1\right\},
$$

and therefore $M$ reduces $T$.

As above,

$$
M=v\left\{T^{*}{ }^{n} P \operatorname{ker} T: n=0,1, \ldots, N-1\right\},
$$

where $P$ is the projection onto $M$. Since $S$ is contained in both $M$ and the kernel of $T$, we have that $S=P S \subseteq P$ ker $T \subseteq \operatorname{ker} T$. It follows that $P$ ker $T \Theta S$ is contained in both $M$ and $H \Theta M$, and consequently $S=P \operatorname{ker} T$.

COROLLARY 2.3. Let $T=\sum_{j=1}^{N} \oplus \hat{T}_{j}$, where $\hat{T}_{j}$ is a generalized truncated shift of index $j$. A subspace $M$ reduces $T$ if and only if $M=\sum_{j=1}^{N} \oplus M_{j}$, where $M_{j}$ reduces $\hat{T}_{j}$.

Proof. Clearly every subspace of the form $\sum_{j=1}^{N} \oplus M_{j}$, where $M_{j}$ reduces $\hat{T}_{j}$, reduces $T$.

Let $M$ reduce $T$ and let $P$ denote the projection onto $M$. Fix $j$ $(I \leq j \leq N)$. By the representation of $T$ and the definition of generalized truncated shift, it follows that 
$\operatorname{ker} \hat{T}_{j}=\operatorname{ker} T \cap \operatorname{ker} T^{* j} \cap \operatorname{range} T^{j-1}$

Since $P$ commutes with $T^{i}$ and $T^{*}$ for every $i=1,2, \ldots, j$, we have that $S=P \operatorname{ker} \hat{T}_{j}$ is contained in $\operatorname{ker} \hat{T}_{j}$ and is invariant under $\hat{T}_{j}^{k} \hat{T}_{j}^{*} k$ for every $k=1,2, \ldots, j-1$. Therefore by Theorem 2.2 , if $H_{j}$ is the domain of $\hat{T}_{j}$, then $M_{j}=\sum_{i=0}^{j-1} \oplus \hat{T}_{j}^{*} i_{S}$ reduces $\hat{T}_{j}$, and

$$
H_{j} \Theta M_{j}=\sum_{i=0}^{j-1} \oplus \hat{T}_{j}^{*}\left(\operatorname{ker} \hat{T}_{j} \Theta S\right)
$$

Since $j$ was arbitrary $(I \leq j \leq N)$, we thus conclude that $M=\sum_{j=1}^{N} \oplus M_{j}$, where $M_{j}$ reduces $\hat{T}_{j}$.

COROLLARY 2.4. Let $T=D T$. be a weighted truncated shift of index $N$ defined on $H=H_{0} \oplus H_{0} \oplus \ldots \oplus H_{0}$ for some Hilbert space $H_{0}$, where $D=\left(A_{1}, A_{2}, \ldots, A_{N-1}, 0\right)$ and $A_{j}$ is one-to-one for every $j=1,2, \ldots, N-1 . A$ subspace $M$ reduces $T$ if and only if

$$
M=S \oplus \sum_{n=1}^{N-1} \oplus v\left\{\left(\prod_{1}^{n} A_{j}\right)^{*} f: f \in S\right\}
$$

for some unique subspace $S$ of $H_{0}$ which is invariant under $\left(\prod_{1}^{k} A_{j}\right)\left\{\prod_{1}^{k} A_{j}\right\}^{*}$ for every $k=1,2, \ldots, N-1$. In this case

$$
H \ominus M=\left(H_{0} \ominus S\right) \oplus \sum_{n=1}^{N-1} \oplus v\left\{\left(\prod_{1}^{n} A_{j}\right\}^{*} f: f \in H_{0} \ominus s\right\}
$$

Proof. A direct computation using Theorem 2.2.

REMARK 2.1. In Corollary 2.4, if $A_{j}$ is invertible for every $j=1,2, \ldots, N-1$, then $\left(\prod_{1}^{n} A_{j}\right)^{*} S$ and $\left(\prod_{1}^{n} A_{j}\right)^{*}\left(H_{0} \Theta S\right)$ are closed for every $n=1,2, \ldots, N-1$. 
REMARK 2.2. In Corollary 2.4, if $A_{j}$ is one-to-one and hermitian for every $j=1,2, \ldots, N-1$, then an induction argument shows that

$$
\left(\prod_{1}^{n} A_{j}\right)^{*} S=S \text { and }\left(\prod_{1}^{n} A_{j}\right)^{*}\left(H_{0} \Theta S\right)=H_{0} \Theta S
$$

for every $n=1,2, \ldots, N-1$. In this case, the conditions $\left(\prod_{1}^{k} A_{j}\right)\left(\prod_{1}^{k} A_{j}\right)^{*} S \subseteq S$ for every $k=1,2, \ldots, N-1$ are equivalent to $A_{j} S \subseteq S$ for every $j=1,2, \ldots, N-1$.

Theorem 2.2 may be modified to hold for the case $N=\infty$. As in Corollary 2.4 this case includes the usual weighted shifts with one-to-one operator weights: if $\left\{A_{1}, A_{2}, \ldots\right\}$ is a uniformly bounded sequence of operators on a complex Hilbert space $\mathcal{C}$, the weighted backward shift $W$ with weights $A_{1}, A_{2}, \ldots$ on the Hilbert space $H^{2}(\mathcal{C})=\mathcal{C} \oplus \mathcal{C} \oplus \ldots$ of all square-summable sequences $\left\{a_{j}\right\}_{j=0}^{\infty}, a_{j}$ in $C$, with norm $\left\|\left\{a_{j}\right\}\right\|^{2}=\Sigma\left|a_{j}\right|^{2}$, is defined by $W\left(a_{0}, a_{1}, \ldots\right)=\left(A_{1} a_{1}, A_{2} a_{2}, \ldots\right)$ ([9], [10]). When $A_{j}=I$ for every $j=1,2, \ldots, W$ is called the unilateral backward shift and will be denoted $W=U_{+}^{*}$.

By a natural extension of Corollary 2.4 and Remarks 2.1 and 2.2 we have the following consequences.

COROLLARY 2.5 (Lambert [9]). Let $W$ be a weighted backward shift on $H^{2}(C)$ with invertible weights $A_{1}, A_{2}, \ldots$. A subspace $M$ of $H^{2}(C)$ reduces $W$ if and only if $M=S \oplus \sum_{n=1}^{\infty} \oplus\left(\prod_{j=1}^{n} A_{j}\right)^{*} S$ for some unique subspace $s$ of $\mathcal{C}$ which is invariant under $\left(\prod_{1}^{k} A_{j}\right)\left(\prod_{1}^{k} A_{j}\right)^{*}$ for every $k=1,2, \ldots$. In this case

$$
H \Theta M=\left(H_{0} \Theta S\right) \oplus \sum_{n=1}^{\infty} \oplus\left(\prod_{j=1}^{n} A_{j}\right)^{*}\left(H_{0} \Theta S\right) \text {. }
$$

COROLLARY 2.6 (Nikol'skii [10]). Let $W$ be a weighted backward 
shift on $H^{2}(C)$ with one-to-one, hermitian weights $A_{1}, A_{2}, \ldots . A$ subspace $M$ of $H^{2}(C)$ reduces $W$ if and only if $M=\sum_{0}^{\infty} \oplus S$ for some unique subspace $S$ of $C$ which is invariant under $A_{1}, A_{2}, \ldots$.

\section{Operators with closed range}

In this section the structure of partial isometries with no isometric part whose first $N+1$ positive integral powers are partial isometries as obtained in [5] will be extended to certain operators whose first $N+1$ powers have closed range. The relationship of these results to partial isometries will be determined in the next section. We begin by establishing a technical lemma for these operators.

LEMMA 3.1. The following are equivalent for an operator $T$ with closed range:

(1) the kernel of $T$ is invariant under $T^{j} T^{* j}$ for every $j=1,2, \ldots, N$;

(2) the kernel of $T^{j}$ is invariant under $T T^{*}$ for every $j=1,2, \ldots, N$;

(3) the image $T^{* j-I}$ ker $T$ is invariant under $T T^{*}$ for every $j=1,2, \ldots, N$.

In this case $T^{2}, T^{3}, \ldots, T^{N+1}$ have closed ranges, and the kermel of $T^{j+1}$, for every $j=1,2, \ldots, N$, is the orthogonal direct sum of the subspaces $T^{* i}$ ker $T \quad(i=0,1, \ldots, j)$.

Proof. (1) implies (2). By induction assume that $T^{j} T^{* j}$ ker $T \subseteq \operatorname{ker} T, T^{j}$ has closed range, $T^{*^{j-1}}$ ker $T$ is closed, $\operatorname{ker} T^{j}=\sum_{i=0}^{j-1} \oplus T^{*} \operatorname{ker} T$, and $T^{* *} \operatorname{ker} T^{j-1} \subseteq \operatorname{ker} T^{j-1} \quad$ for every $j=1,2, \ldots, N$. It suffices to show that $T^{N+1}$ has closed range, $T^{*}$ ker $T$ is closed, $\operatorname{ker} T^{N+1}=\sum_{i=0}^{N} \oplus T^{*}$ ker $T$ and 
$T T^{*} \operatorname{ker} T^{N} \subseteq \operatorname{ker} T^{N}$

Let $f$ be in the closure of the range of $T^{*} N+1$. Since $T^{*} *^{N}$ has closed range, $f=T^{*}{ }^{N}$ for some $g$ in $H$. Write $g=T^{*} h+k$ where $h$ is in $H$ and $k$ is in the kernel of $T$. Then, by (1), $T^{N+1} f=T^{N+1} T^{*}{ }^{N+1} h$, so that $f-T^{* N+1} h$ is in both the kernel of $T^{N+1}$ and the closure of the range of $T^{*} N+1$. Therefore $f$ is in the range of $T^{* N+1}$. It follows that $T^{* N+1}$, and consequently $T^{N+1}$, have closed ranges.

Similarly, let $f$ be in the closure of $T^{*^{N}}$ ker $T$. As above, $f=T^{*} g$ where $g$ is in $H$, and if $g=T^{*} h+k$ where $h$ is in $H$ and $k$ is in the kernel of $T$, then $T^{N+1} f=T^{N+1} T^{*}{ }^{N+1} h$. By (I), $T^{N+1} f=0$ and hence $T^{* N+1} h=0$. Therefore $f=T^{*{ }^{N}} k$ is in $T^{*^{N}}$ ker $T$.

Next note that $T^{*^{i}}$ ker $T$ is orthogonal to $T^{*^{j}}$ ker $T$ for all $0 \leq i \neq j \leq N$, and $\sum_{i=0}^{N} \oplus T^{*^{i}}$ ker $T$ is contained in the kernel of $T^{N+1}$ by (1). Let $f$ be in $\operatorname{ker} T^{N+1} \ominus \sum_{i=0}^{N} \oplus T^{*^{i}}$ ker $T$. By assumption $f$ is orthogonal to the kernel of $T^{N}$, so that $f=T^{*} N g$, where $g$ is in $H$, since $T^{* N}$ has closed range. As above, if $g=T^{*} h+k$, where $h$ is in $H$ and $k$ is in the kernel of $T$, then $f=T^{*} N_{k}$. Therefore $f=0$, and $\operatorname{ker} T^{N+1}=\sum_{i=0}^{N} \oplus T^{* i} \operatorname{ker} T$.

Finally since $T T^{*}\left(T^{*}{ }^{N-1} \operatorname{ker} T\right) \subseteq \operatorname{ker} T^{N}$ by (1), and $\operatorname{ker} T^{N}=\operatorname{ker} T^{N-1} \oplus T^{*^{N-1}}$ ker $T$, it follows that $T T^{*} \operatorname{ker} T^{N} \subseteq \operatorname{ker} T^{N}$.

(2) implies (1). By induction assume (2) and $T^{j} T^{* j}$ ker $T \subseteq \operatorname{ker} T$ for every $j=1,2, \ldots, N-1$. Then $T^{*^{N-1}}$ ker $T$ is contained in the kernel of $T^{N}$, and therefore 


$$
T\left(T^{N} T^{*} \text { ker } T\right)=T^{N}\left(T T^{*}\right) T^{* N-1} \operatorname{ker} T \subseteq T^{N} \operatorname{ker} T^{N}=\{0\} .
$$

(2) implies (3). By induction assume (2) and

$$
T T^{*}\left(T^{* j-1} \operatorname{ker} T\right) \subseteq T^{* j-1} \operatorname{ker} T
$$

for every $j=1,2, \ldots, N-1$. Since (2) is equivalent to (1), the above shows that $T^{*}$ ker $T$ is closed for every $i=0,1, \ldots, N-1$, and $\operatorname{ker} T^{N}=\sum_{i=0}^{N-1} \oplus T^{*^{i}} \operatorname{ker} T$. Moreover by (2), TT* $\left(T^{*} N-1\right.$ ker $\left.T\right) \subseteq \operatorname{ker} T^{N}$. Thus since $T T^{*}\left(T^{* N-1} \operatorname{ker} T\right)$ is orthogonal to $T^{* i}$ ker $T$ for every $i=0,1, \ldots, N-2$, it follows that $T T^{*}\left(T^{*}{ }^{N-1}\right.$ ker $\left.T\right)$ is contained in $T^{* N-1} \operatorname{ker} T$.

(3) implies (1). An immediate consequence of (3) and the identity $T^{j} T^{* j}=T^{j-1}\left(T T^{*}\right) T^{* j-1}$.

THEOREM 3.1. A necessary and sufficient condition that $T$ be an operator on Hilbert space with closed range such that the kernels of $T^{j}$ and $T^{* j}$ are invariant under $T T^{*}$ and $T^{*} T$ respectively for every $j=1,2, \ldots, N$ is that $T=\hat{T}_{1} \oplus \hat{T}_{2} \oplus \ldots \oplus \hat{T}_{N} \oplus V$ where $\hat{T}_{j}$ is a generalized truncated shift of index $j$ and $V$ is an operator with closed range such that $V^{*}\left(\operatorname{ker} V^{j}\right)=\operatorname{ker} V^{j}$ and $V^{*} V\left(\operatorname{ker} V^{* j}\right)=\operatorname{ker} V^{* j}$ for every $j=1,2, \ldots, N$. Moreover, the representation so expressed is unique, and a projection $P$ commutes with $T$ if and only if $P=P_{1} \oplus P_{2} \oplus \ldots \oplus P_{N} \oplus Q$ where $P_{j}$ and $Q$ are projections which commute with $\hat{T}_{j}$ and $V$ respectively $(j=1,2, \ldots, N)$.

Proof. Sufficiency follows directly from Corollary 2.1, Lemma 3.1, and the definition of generalized truncated shift.

To show necessity let $c_{j}=\operatorname{ker} T \cap T^{j-1}$ ker $T^{*}$ for every $j=1,2, \ldots, N$. Since the kernel of $T^{*}$ is invariant under $T^{*^{j-1}} T^{j-1}$ by Lemma 3.1, we have that $C_{j}=\operatorname{ker} T \cap$ ker $T^{*^{j}} \cap$ range $T^{j-1}$ for every $j=1,2, \ldots, N$. 
The linear manifold $T^{*}{ }^{i-1} C_{j}$ is invariant under $T T^{*}$ for all $0<i<j \leq N$ : to verify this, fix $i$ and $j(0<i<j \leq N)$ and let $f=T^{j-1} g$ be in $C_{j}$ where $g$ is in the kernel of $T^{*}$. Since $T^{k}$ ker $T^{*}$ is invariant under $T^{*} T$ for every $k=1,2, \ldots, j-2$ by Lemma 3.1, $T T^{*} f=T T^{*}{ }^{i-1}\left(T^{*} T\right) T^{j-2} g$ is in the kernel of $T^{* j-i+1}$. Furthermore since $T^{*}{ }^{i-1}$ ker $T$ is invariant under $T T^{*}$ by Lemma 3.1, $T T^{*}{ }^{i} f$ is in $T^{*}{ }^{i-1}\left(\operatorname{ker} T \cap \operatorname{ker} T^{*^{j}}\right)$. Therefore $T T^{*^{i}} f$ is in $T^{*^{i}-1} \mathcal{C}_{j}$, since the kernel of $T^{* j}$ is the orthogonal direct sum of the subspaces $T^{k}$ ker $T^{*}$ $(k=0,1, \ldots, j-1)$ and $T T^{*^{i}} f$ is orthogonal to $T^{*^{i-1} T^{k}}$ ker $T^{*}$ for all $k<j-1$.

Let $H_{j}$ be the closed span of the images $T^{*}{ }^{i} C_{j}(i=0,1, \ldots, j-1)$ for every $j=1,2, \ldots, N$. Fix $j(1 \leq j \leq N)$. Then $H_{j}$ reduces $T$ by the above, and $C_{j}$ is the kernel of $T$ restricted to $H_{j}$. Hence, by Lemma 3.1, $T^{*} C_{j}$ is closed, and since $T T^{*}$ has closed range and has $T^{*}{ }^{i-1} C_{j}$ as an invariant subspace, it follows that $T T^{*}\left(T^{*}{ }^{i-1} C_{j}\right)$ is closed for every $i=1,2, \ldots, j-1$. Since $T T^{*} C_{j}$ is dense in $T^{* i-1} C_{j}$, we have that $T T{ }^{*} C_{j}=T^{*}{ }^{i-1} C_{j}$ for every $i=1,2, \ldots, j-1$. Therefore, since $j$ was arbitrary, the restriction of $T$ to $H_{j}$ is a generalized truncated shift $\hat{T}_{j}$ of index $j$ for every $j=1,2, \ldots, N$.

Since the kernel of $T$ is invariant under $T^{j-1} T^{* j-1}$ by Lemma 3.1, it follows that

$$
C_{j}=\left(\operatorname{ker} T \cap \operatorname{ker} T^{* j}\right) \ominus\left(\operatorname{ker} T \cap \operatorname{ker} T^{* j-1}\right)
$$

for every $j=1,2, \ldots, N$. Consider the restriction $V$ of $T$ to the orthogonal complement of $\sum_{j=1}^{N} \oplus H_{j}$. Clearly $V^{*} V$ ker $V^{*^{j}} \subseteq \operatorname{ker} V^{*^{j}}$ and 
$V V^{*} \operatorname{ker} V^{j} \subseteq \operatorname{ker} V^{j}$ for every $j=1,2, \ldots, N$. Thus, since ker $V=\operatorname{ker} T \cap$ range $T^{N}$, the image $V^{* j}$ ker $V$ is contained in the range of $V$ for every $j=0,1, \ldots, N-1$. Therefore $\operatorname{ker} V^{j} \Theta V V^{*} \operatorname{ker} V^{j}$ is contained in both the kernel of $V^{*}$ and the kernel of $V^{j}$, and consequently ker $V^{j}=W^{*}$ ker $W^{j}$ for every $j=1,2, \ldots, N$. Similarly ker $V^{* j}=V^{*} V$ ker $V^{* j}$ for every $j=1,2, \ldots, N$, since the images $V^{j}$ ker $V^{*}(j=0,1, \ldots, N-1)$ are contained in the range of $V^{*}$. Next, let $M$ reduce $T=\sum_{j=1}^{N} \oplus \hat{T}_{j} \oplus V$ and let $P$ be the projection onto $M$. By the above construction,

$$
\sum_{j=1}^{N} \oplus H_{j}=\sum_{j=0}^{N-1} \oplus T^{*^{j}}\left(\operatorname{ker} T \cap \operatorname{ker} T^{*^{N}}\right)
$$

Since $P$ commutes with $T$ and $T^{*} N$, we have that $\operatorname{ker} T \cap$ ker $T^{*}$ is invariant under $P$. Therefore

$$
P \sum_{j=1}^{N} \oplus H_{j}=\sum_{j=0}^{N-1} \oplus T^{* j} P\left(\operatorname{ker} T \cap \operatorname{ker} T^{*}{ }^{N}\right)
$$

is contained in $\sum_{j=1}^{N} \oplus H_{j}$, and thus $M=\hat{M} \oplus N$, where $\hat{M}$ reduces $\sum_{j=1}^{N} \oplus \hat{T}_{j}$ and $N$ reduces $V$. The desired form of $P$ now follows from Corollary 2.3.

Finally, uniqueness is a direct consequence of the explicit nature of the above construction.

REMARK 3.1. In the above theorem, it follows from Lemma 3.1 that $T=V$ if and only if, in addition to the invariance conditions on $T$, the kernel of $I^{*}$ is orthogonal to the kernel of $T^{j}$ for every $j=1,2, \ldots, N$.

REMARK 3.2. For an operator $V$ with closed range, the conditions $V^{*} \operatorname{ker} V^{j}=\operatorname{ker} V^{j}$ and $V^{*} V \operatorname{ker} V^{* j}=\operatorname{ker} V^{* j}$ for every 
$j=1,2, \ldots, N$ are equivalent to $V^{j} V^{* j}$ ker $V=\operatorname{ker} V$ and $V^{*} j^{j}$ ker $V^{*}=\operatorname{ker} V^{*}$ for every $j=1,2, \ldots, N$. In the next section, these conditions will be simplified if $V$ is a partial isometry.

Theorem 2.1 and the following result relate the decomposition in Theorem 3.1 to partial isometries. We recall that every operator $V$ on Hilbert space has the polar decomposition $V=A W$ where $A=\left(V V^{*}\right)^{\frac{1}{2}}$ and $W$ is a partial isometry with initial set the orthogonal complement of the kernel of $V$ and final set the closure of the range of $V$ [7].

PROPOSITION 3.1. Let $V$ be an operator with closed range such that $V V^{*}\left(\operatorname{ker} V^{j}\right)=\operatorname{ker} V^{j}$ for every $j=1,2, \ldots, N$. Then the partial isometry $W$ in the polar decomposition $V=A W$ of $V$ satisfies $W W^{*} \operatorname{ker} W^{N}=\operatorname{ker} W^{N}$ and therefore $W^{2}, w^{3}, \ldots, w^{N+1}$ are partial isometries.

Proof. By induction assume that $V^{*}\left(\operatorname{ker} V^{j}\right)=\operatorname{ker} V^{j}$ for every $j=1,2, \ldots, N, W W^{*} \operatorname{ker} W^{N-1}=\operatorname{ker} W^{N-1}$, and $\operatorname{ker} W^{N-1}=\operatorname{ker} V^{N-1}$. By Lemma 3.1, $\operatorname{ker} V^{j}=\operatorname{ker} V^{j-1} \oplus V^{* j-1} \operatorname{ker} V$ and

$$
\operatorname{ker} w^{j}=\operatorname{ker} W^{j-1} \oplus W^{* j-1} \operatorname{ker} W
$$

for every $j=1,2, \ldots, N$. Now $\operatorname{ker} V=\operatorname{ker} W$ and

$$
\begin{aligned}
& W^{N}\left(V^{*}{ }^{N-1} \operatorname{ker} V\right)=W^{N-1}\left(W V^{*}\right) V^{*} \text { ker } V \\
& \subseteq W^{N-1} A \text { ker } V^{N-1} \subseteq W^{N-1} \operatorname{ker} V^{N-1}=W^{N-1} \operatorname{ker} W^{N-1}=\{0\} \text {, }
\end{aligned}
$$

since $A$ is the strong limit of a sequence of polynomials in $V V^{*}$. Thus we have that ker $V^{N} \subseteq$ ker $W^{N}$. Similarly ker $W^{N} \subseteq$ ker $V^{N}$. Therefore $W W^{*} \operatorname{ker} W^{N}=W W^{*} \operatorname{ker} V^{N}=W W^{*}\left(V V^{*} \operatorname{ker} V^{N}\right)=V V^{*} \operatorname{ker} V^{N}=\operatorname{ker} V^{N}=\operatorname{ker} W^{N}$.

Finally $w^{2}, w^{3}, \ldots, w^{N+1}$ are partial isometries by [3, Theorem 2].

REMARK 3.3. In Theorem 3.1, if, in addition to the invariance conditions on $T$, the kernel of $T^{*}$ is contained in the kernel of $T^{N}$, then $V=A W$ where $A=\left(V V^{*}\right)^{\frac{1}{2}}$ and $W^{*}$ is an isometry. 
Let $T$ be an operator on a Hilbert space $H$ and suppose that $T$ has closed range. We recall that the generalized inverse of $T$, denoted by $T^{+}$, is the operator on $H$ defined as follows: if $h=T f+g$ is the unique decomposition of a vector $h$ in $H$, where $f$ is orthogonal to the kernel of $T$ and $g$ is in the kernel of $T^{*}$, then $T^{+} h=f$ [1]. By a straightforward induction argument it follows that condition (1) of Lemma 3.1 implies that the identity $\left(T^{j+1}\right)^{+}=\left(T^{+}\right)^{j+1}$ holds on the range of $T^{j+1}$ for every $j=1,2, \ldots, N$. The next result characterizes those operators satisfying Theorem 3.1 for which this identity holds everywhere.

PROPOSITION 3.2. Let $T$ be an operator such that $T^{N+1}$ has closed range for some positive integer $N$ and let $E=T\left(T^{N+1}\right)^{+} T^{N}$. If $\|E\| \leq 1$, then $T T^{*} \operatorname{ker} T^{N} \subseteq \operatorname{ker} T^{N}$. Conversezy, if $T T^{*}$ ker $T^{N} \subseteq$ ker $T^{N}, T$ and $T^{N}$ have closed ronge, $\left(T^{N+1}\right)^{+}=T^{+}\left(T^{N}\right)^{+}$, and $T^{*} T^{N}$ ker $T^{*} \subseteq$ ker $T^{*}$, then $\|E\| \leq 1$.

Proof. Assume $\|E\| \leq 1$. Since $E^{2}=E$ it follows that $E$ is hermitian. Let $f$ be in the kernel of $T^{N}$. Then $T^{*}\left(T^{*} N+1\right) T^{*} f=0$, and hence $\left(T^{* N+1}\right){ }^{*} f=0$. Therefore $T^{*} f$ is in the kernel of $T^{N+1}$. Since $f$ was arbitrary, $T T^{*}$ ker $T^{N} \subseteq$ ker $T^{N}$.

For the converse, note that $E=E^{*}=0$ on the kernel of $T^{N}$. Let $f$ be orthogonal to the kernel of $T^{N}$. Then

$$
E f=\left(T T^{+}\right)\left(T^{N^{+}} T^{N}\right) f=T T^{+} f
$$

and

$$
E^{*} f=\left(T^{*}\left(T^{*}\right)^{+}\right)\left(T^{*} T^{*}\right) f=\left(T^{N^{+}} T^{N}\right)\left(T T^{+}\right) f
$$

Since $T^{* N^{N}} T^{N}$ ker $T^{*} \subseteq$ ker $T^{*}$ and $T T^{+} f$ is the projection of $f$ onto the range of $T$, it follows that $T T^{+} f$ is orthogonal to the kernel of $T^{N}$, and consequently $E^{*} f=E f$. Therefore $E$ is hermitian and idempotent, and thus $\|E\| \leq 1$.

PROPOSITION 3.3. Let $T$ be a contraction with closed range such 
that $T^{2}, T^{3}, \ldots, T^{N+1}$ are partial isometries. Then $T$ satisfies the hypotheses of Theorem 3.1 .

Proof. Since $\left(T^{j+1}\right)^{+}=T^{* j+1}$ for every $j=1,2, \ldots, N$ and $\|T\| \leq 1$, this result follows immediately from Proposition 3.2.

\section{Power partial isometries}

Partial isometries on Hilbert space all of whose positive integral powers are partial isometries were introduced and characterized in [8]. These results were extended in [5] to partial isometries $T$ whose first $N+1$ powers are partial isometries under the assumption that $T$ has no isometric part. A direct consequence of the previous sections and of the following lemma makes this assumption unnecessary.

LEMMA 4.1. The following are equivalent for a partial isometry $V$ :

(1) $V V^{*} \operatorname{ker} V^{N}=\operatorname{ker} V^{N}$ for some positive integer $N$;

(2) the image $V^{* j-1}$ (ker $V$ ) is contained in the range of $V$ for every $j=1,2, \ldots, N$.

(3) $V^{j} V^{* j}$ ker $V=\operatorname{ker} V$ and $V^{* j} V^{j}$ ker $V^{*}=\operatorname{ker} V^{*}$ for every $j=1,2, \ldots, N$.

In this case, $v^{2}, v^{3}, \ldots, v^{N+1}$ are partial isometries.

Proof. (1) implies (2). By (1), since $V$ is a partial isometry, $V V^{*}=I$ on the kernel of $V^{j}$ for every $j=1,2, \ldots, N$, and hence by Lemma 3.1, $\operatorname{ker} V^{N}=\sum_{j=0}^{N-1} \oplus V^{*^{j}}$ ker $V$. Therefore $V^{* j-1}($ ker $V) \subseteq$ range $V$ for every $j=1,2, \ldots, N$.

(2) implies (3). An immediate consequence of (2) and the identities $V^{j} V^{* j}=V^{j-1}\left(V V^{*}\right) V^{* j-1}$ and $V^{* j} V^{j}=V^{* j-1}\left(V^{*} V\right) V^{j-1}$.

(3) implies (1). By Lemma 3.1, ker $V^{N}=\sum_{j=0}^{N-1} \oplus V^{* j} \operatorname{ker} V$, and by [3, Theorem 2], $v^{2}, v^{3}, \ldots, v^{N+1}$ are partial isometries. Fix $j$ 
$(0 \leq j \leq N-1)$. Let $f$ be in $V^{* j}$ ker $V$. Then since $V^{j} V^{j}$ is the projection onto the range of $V^{j}$, we have that $f=V^{* j} g$ where $g=V^{j} V^{j} g=V^{j} f$ is in the kernel of $V$. Moreover, $V^{j}\left(V V^{*}\right) f=V^{j+1} V^{* j+1} g=g=V^{j} f$. Therefore $\left(1-V V^{*}\right) f$ is in both the kernel of $V^{j}$ and the kernel of $V^{*}$, and consequently, by (3), $W^{*} f=f$. Since $f$ and $j$ were arbitrary, we conclude that $V^{*} \operatorname{ker} V^{N}=\operatorname{ker} V^{N}$.

The following theorem is a consequence of Proposition 3.3, Theorem 3.1, Corollary 2.2, and Lemma 4.1.

THEOREM 4.1. A necessary and sufficient condition that $T$ be a partial isometry on Hilbert space such that $T^{2}, T^{3}, \ldots, T^{N+1}$ are partial isometries is that $T=T_{1} \oplus T_{2} \oplus \ldots \oplus T_{N} \oplus V$ where $T_{j}$ is a truncated shift of index $j$ and $V$ is a partial isometry such that the kernel of $V^{N}$ is contained in the ronge of $V$. Moreover, this representation is unique, and a projection $P$ commutes with $T$ if and only if $P=P_{1} \oplus P_{2} \oplus \ldots \oplus P_{N} \oplus Q$ where $P_{j}$ and $Q$ are projections which commite with $T_{j}$ and $V$ respectively $(j=1,2, \ldots, N)$.

Theorems 3.1 and 4.1 have natural extensions to the case $N=\infty$ as the following result indicates.

COROLLARY 4.1 (Halmos-Wallen). $T^{j}$ is a partial isometry on Hilbert space for every $j=1,2, \ldots$ if and only if $T=\left(\sum_{I}^{\infty} \oplus T_{j}\right) \oplus U_{+1}^{*} \oplus U_{+2} \oplus U$, where $T_{j}$ is a truncated shift of index $j, U_{+}$is a unilateral shift, and $U$ is unitary. Moreover, the representation so expressed is unique.

Proof. The proof follows from Theorem 4.1 as in the proof of [5, Corollary 3.2].

COROLLARY 4.2 (Fishel [4]). Let $T$ be a partial isometry on Hilbert space. Then $T=U_{+1}^{*} \oplus U_{+2} \oplus U$ unique $z_{\text {s }}$ where $U_{+i}$ is a unilateral shift and $U$ is unitary, if and only if the kermel of $T^{*}$ is orthogonal to the kernel of $T^{j}$ for every $j=1,2, \ldots$.

Proof. Lemma 4.1, Theorem 4.1, Remark 3.1, and Corollary 4.1. 
COROLLARY 4.3. Let $T$ be a partial isometry on Hilbert space. Then $T=T_{1} \oplus T_{2} \oplus \ldots \oplus T_{N} \oplus U_{+}^{*} \oplus U$ unique $z_{y}$ where $T_{j}$ is a truncated shift of index $j, U_{+}$is a unilateral shift, and $U$ is unitams if and only if $T^{2}, T^{3}, \ldots, T^{N+1}$ are partial isometries and the kermel of $T^{*}$ is contained in the kermel of $T^{N}$.

Proof. Theorem 4.1, Remark 3.3, and Corollary 4.1.

\section{References}

[1] Adi Ben-Israel, Thomas N.E. Greville, Generalized inverses: theory and applications (John Wiley \& Sons, New York, London, Sydney, Toronto, 1974).

[2] Louis de Branges, James Rovnyak, "Canonical models in quantum scattering theory", Perturbation theory and its opplications in quantum mechanics, 295-392 (Proc. Adv. Sem. Math. Res. Center, U.S. Army, Theoret. Chem. Instit., University of Wisconsin, Madison, 16. John Wiley \& Sons, New York, London, Sydney, 1966).

[3] I van Erdelyi, "Partial isometries closed under multiplication on Hilbert spaces", J. Math. Anal. Appl. 22 (1968), 546-551.

[4] B. Fishel, "Partial isometries which are sums of shifts", Math. Proc. Cambridge Philos. Soc. 78 (1975), 107-110.

[5] James Guyker, "On partial isometries with no isometric part", Pacific J. Math. 62 (1976), 419-433.

[6] James Guyker, "Reducing subspaces of contractions with no isometric part", Proc. Amer. Math. Soc. 45 (1974), 411-413.

[1] Paul R. Halmos, A Hilbert space problem book (Van Nostrand, Princeton, New Jersey; Toronto; London; 1967).

[8] P.R. Halmos \& L.J. Wallen, "Powers of partial isometries", J. Math. Mech. 19 (1970), 657-663.

[9] Alan Lambert, "Unitary equivalence and reducibility of invertibly weighted shifts", Bulz. Austral. Math. Soc. 5 (1971), 157-173. 
[10] Н.H. Нинольгчнй [N.K. Nikol'skiY]], "Об ннөарнантных подпространствах взеешенных операторов сдвига" [Invariant subspaces of weighted shift operators], Mat. Sb. (II.S.) 74 (116) (1967), 171-190. English Transl.: Math. USSR Sb. 3 (1967), 159-176.

Department of Mathematics, State University of New York,

College at Buffalo,

Buffalo,

New York,

USA. 